\title{
DE POLÍTICAS Y SUBJETIVIDADES. NUEVAS \\ ESTRATEGIAS DE INTERVENCIÓN EN DISPOSITIVOS DE \\ INCLUSIÓN SOCIO-EDUCATIVA Y LABORAL PARA \\ JÓVENES EN ARGENTINA (2008-2016)
}

\author{
POLICIES AND SUBJECTIVITIES. NEW STRATEGIES OF \\ INTERVENTION IN DEVICES INCLUDING SOCIO-EDUCATIONAL \\ AND LABOR FOR YOUTH IN ARGENTINA (2008-2016)
}

http://dx.doi.org/10.22235/pe.v9i2.1295

Recibido: 16-08-2016

Revisado: 17-09-2016

Aceptado: 18-09-2016

\section{SABRINA FERRARIS \\ EUGENIA ROBERTI ${ }^{*}$}

ALEJANDRO BURGOS ${ }^{* *}$

JOSÉ ANTONIO POZZER ${ }^{* \star \star}$

\footnotetext{
* Licenciada en Sociología y doctora en Ciencias Sociales (Universidad de Buenos Aires), especialista en Demografía Social (Universidad Nacional de Luján). Docente en la Universidad de Buenos Aires y becaria posdoctoral de CONICET en el Instituto Interdisciplinario de Economía Política de Buenos Aires (IIEP BAIRES). Ha escrito sobre temáticas referidas a transiciones a la vida adulta, trayectorias laborales y juventudes en Argentina, así como estudios de masculinidad y el rol de proveedor de los hombres en México.
}

** Licenciada en Sociología, magíster en Ciencias Sociales y doctoranda en Ciencias Sociales (Universidad Nacional de La Plata). Becaria doctoral del Consejo Nacional de Investigaciones Científicas y Técnicas (CONICET-CIS/IDES) y docente en la FaHCE-UNLP. Sus principales temáticas de interés son los jóvenes, las políticas públicas y los estudios de trayectorias.

*** Licenciado en Educación (Universidad Nacional de General Sarmiento). Magíster en Educación: Pedagogías Críticas y Problemáticas Socioeducativas (Universidad de Buenos Aires). Doctorando en el Doctorado en Ciencias Sociales (Universidad de Buenos Aires). Becario doctoral del Consejo Nacional de Investigaciones Científicas y Técnicas (CONICET-CIS/IDES). Sus temas de investigación son la desigualdad educativa, la inclusión social mediante dispositivos de formación para el trabajo y educación, y los estudios de trayectorias en jóvenes y adultos.

**** Licenciado en Relaciones Laborales (Universidad Nacional del Nordeste). Doctorando en Ciencias Sociales (Universidad Nacional de Entre Ríos). Becario Interno doctoral del CONICET con sede en la Facultad de Ciencias Económicas (UNNE) y docente de dicha unidad académica. Miembro del PREJET, estudia los 
Resumen: El trabajo se propone aportar a los estudios que complejizan el análisis de las transiciones entre educación y trabajo. Así, se analizan dispositivos de inclusión socio-educativa y laboral que pretenden intervenir en la subjetividad de las personas que participan de ellos, en pos de mejorar sus oportunidades de inclusión socio-laboral. Se presentan dos casos con particularidades propias: el Programa Jóvenes con Más y Mejor Trabajo y un Centro de Formación Profesional, ambos radicados en dos municipios del conurbano bonaerense de la Argentina. Los casos se abordan desde una doble mirada: la institucional, a través de la normativa que los regula y las voces de sus gestores, y el discurso de los propios sujetos participantes.

Palabras clave: Políticas sociales, estrategias institucionales, inclusión social, subjetividades

\begin{abstract}
The paper aims to contribute to studies that complicate the analysis of transitions between education and work. Thus, are analyzed devices including socio-educational and job seeking to intervene in the subjectivity of people who participate in them, after improving opportunities for socio-labor inclusion. Two cases are presented, with particular features: The Youth Program for More and Better Jobs and a Vocational Training Center, both based in two municipalities of Argentina. The cases are approached from a double perspective: institutional through the regulations governing them and the voices of their managers, and the speech of participants subjects themselves.
\end{abstract}

Keywords: Social politics, institutional strategies, social inclusion, subjectivities

procesos de socialización laboral de jóvenes mediante dispositivos de formación e inserción para el trabajo en la ciudad de Resistencia (Chaco). 


\section{INTRODUCCIÓN}

Este artículo se enmarca en las investigaciones que, en las últimas décadas, señalan la importancia de incorporar la dimensión de las subjetividades en los estudios sobre educación y trabajo, específicamente en las intervenciones institucionales que se generan para acompañar las transiciones al mercado laboral de los jóvenes.

Estudios previos muestran la compleja trama de dimensiones estructurales, institucionales e individuales que se entretejen en los cursos de vida de los jóvenes, lo que conlleva múltiples transiciones juveniles. Ante el debilitamiento y los cambios en las instituciones que tradicionalmente acompañaron dicho proceso -fundamentalmente la familia y la escuela - aparecen otros soportes que pretenden intervenir, muchos de los cuales hacen hincapié en actuar sobre las subjetividades. Así, las preguntas que guían este artículo son: ¿qué estrategias de intervención sobre las subjetividades proponen los dispositivos de educación y empleo dirigidos a jóvenes? y ¿qué respuestas desarrollan y generan los propios jóvenes?

Para abordar esta problemática se presentan, en primer lugar, los debates centrales en torno a los estudios sobre la relación entre educación y trabajo, poniendo el eje en aquellos que valorizan la dimensión de las subjetividades. En segundo lugar, se analizan dos casos de intervenciones institucionales que permiten reflexionar sobre los soportes subjetivos desarrollados para mejorar las oportunidades de los jóvenes: el Programa Jóvenes con Más y Mejor Trabajo y un Centro de Formación Profesional, ambos radicados en dos municipios del conurbano bonaerense de la Argentina.

Estos dispositivos resultan interesantes a la hora de desentrañar los procesos de subjetivación que proponen, ya sea desde las estrategias de intervención generadas por las instituciones y gestionadas por sus referentes, como también desde el desarrollo de valoraciones, opiniones y prácticas por parte 
de los propios jóvenes que participan. De ese modo, y sin pretender comparaciones entre los casos, se abordan dos niveles de análisis: el propuesto por el dispositivo (revisión de la normativa y discursos de los gestores) y el discurso de los participantes.

\section{LA COMPLEJIDAD EN LAS TRANSICIONES EDUCACIÓN-TRABAJO: ESTRUCTURA, BIOGRAFÍAS Y SUBJETIVIDADES}

La relación entre educación y trabajo ha sido investigada desde diversos enfoques. En las últimas dos décadas del siglo XX, como señala Jacinto (2010a), el aumento generalizado del desempleo, en particular en los jóvenes, puso de manifiesto el límite de las teorías basadas en una concepción mecánica y simplificadora de dicha relación, como la teoría del capital humano. Diversos aportes han señalado estas supuestas inadecuaciones (Bourdieu y Passeron, 1967, 1972; Arrow, 1973; Carnoy, 1982), vinculadas tanto a la expansión del número de egresados del sistema educativo como también a la crisis del empleo.

Asimismo, desde la sociología de la juventud -a partir de las transformaciones en los tiempos juveniles - se ha evidenciado que el par educación-trabajo no responde a un modelo lineal, puesto que a veces estos procesos se superponen o bien sus secuencias son complejas y poco previsibles (Galland, 2002).

En épocas más recientes, desde la sociología del trabajo, comienzan a cobrar fuerza investigaciones que dan cuenta -a través de los estudios sobre socialización profesional y construcción de identidades profesionales en contextos de precarización laboral (Dubar, 1991; Nicole-Drancourt y Roulleau-Berger, 2001) - de cómo los procesos de individualización condicionan fuertemente las trayectorias de inserción de los jóvenes (Jacinto, 2010a).

Ahora bien, estas tendencias de estudios sobre la inserción laboral con relación a la educación suceden en un contexto histórico singular que trasciende el caso argentino. La mutación de las normas de empleo hacia un modelo más flexible, inestable y precario (Castel y Haroche, 2001; Dubar, 2002; Salvia y 
Tuñón, 2003a; Pérez, 2008; Longo, 2010) está acompañada de un debilitamiento de las instituciones antaño centrales para la socialización —la familia, la escuela, el empleo y los sindicatos- (Dubet, 2002) y de una transformación de las temporalidades sociales y biográficas, que destacan la incertidumbre (Hartog, 2003; Beck, 2008). Los seres humanos se sumergen en una búsqueda constante por intentar encontrar "soluciones biográficas" a contradicciones sistémicas de un contexto inestable y cambiante (Beck, 2008). Todos estos fenómenos coadyuvan, en definitiva, a cuestionar la linealidad en las transiciones juveniles.

Como señala Longo (2008), esto lleva a preguntarse no solo por las transiciones y sus procesos, sino también por las herramientas sociológicas para observarlas y por los dispositivos políticos para regularlas. En este sentido, la autora apunta que una de las dificultades en el ámbito de la investigación suele ser la simplificación y la desarticulación de factores, el enfatizar en exceso ciertos aspectos en desmedro de otros menos estudiados.

La complejidad del par educación-trabajo reclama una mayor integralidad de las dimensiones estructurales, institucionales, individuales y subjetivas que condicionan el acceso de los jóvenes a buenos empleos y plantean desafíos específicos a las estrategias de intervención. Desde el ámbito académico son varios los autores que señalan la importancia de un abordaje multidimensional (Casal, 1996; Riquelme, 2001; Walther y Pohl, 2005; Abdala, Jacinto y Solla, 2005; Weller, 2007; Salvia, 2008; Jacinto, 2008; Herger, 2008). Así, han cobrado relevancia estudios sociológicos que, desde un enfoque sistémico, logran integrar los niveles macro y microsociales, al poner como eje las mediaciones institucionales y las subjetividades (Morch, 2002; Jacinto, 2010a).

Una posible lectura de este "rescate" de la dimensión subjetiva, según Longo (2008), es que en un contexto de desinstitucionalización en el que los sujetos deben reconstruir las bases de su identidad —que ya no están dadas a priori-, las relaciones simbólicas y otros factores subjetivos ganan peso y desplazan el interés hacia los protagonistas y a sus compromisos, argumentaciones y construcciones sobre la realidad que los rodea. Sin embargo, 
De políticas y subjetividades. Nuevas estrategias de intervención en dispositivos de inclusión socioeducativa y laboral para jóvenes en Argentina (2008-2016)

algunos trabajos advierten que el proceso de individualización resulta asimétrico en función de los condicionantes económicos y sociales que proveen recursos, expectativas, inhibiciones y habilitaciones (Robles, 2000).

En efecto, las trayectorias reflejan tanto voluntad personal como condicionantes estructurales y contextuales que se retroalimentan y hacen a la diversidad de recorridos vitales. Las experiencias en los cursos de vida $-\mathrm{y}$, en ellas, los sentidos, significaciones, estrategias o elecciones-permiten comprender las singularidades de cada trayectoria (Jacinto, 2007; Jacinto, 2010a). Así, poner el foco en la subjetividad de los jóvenes no solo muestra los condicionantes macro-estructurales adversos, la desafiliación y los cambios en las subjetividades en torno a la educación y el trabajo, sino que también logra captar las dinámicas al considerar la institucionalidad y otras esferas de la vida (Jacinto y Millenaar, 2009; Jacinto, 2010b).

En este contexto reciente, que recupera la subjetividad de los jóvenes en los estudios sobre educación y trabajo, interesa reflexionar sobre cuánto de ello se manifiesta a la hora de implementar políticas en torno a la inserción laboral juvenil. En este sentido, el deterioro de las instituciones tradicionales afecta el proceso de construcción del sujeto, pero no a todos los grupos sociales de la misma manera. En los sectores excluidos, ese deterioro tiene significados asociados con derechos y necesidades no satisfechos de educación, de salud o de protección social, así como un déficit de socialización. Y es por ello que, siguiendo a Tedesco (2008), vale preguntarse por las "políticas de subjetividad" destinadas a garantizar condiciones institucionales que permitan cumplir con el derecho a la subjetividad para todos. La búsqueda de estrategias para fortalecer la autoestima, la capacidad de conocerse a sí mismos y de articular narrativamente sus deseos, demandas y necesidades es lo que contribuye a formar subjetividades (Tedesco, 2008).

\section{De políticas y subjetividades}

Pensar a los jóvenes como sujetos de políticas es un desafío que ha logrado instalarse en la agenda pública desde hace al menos dos décadas y que ha 
De políticas y subjetividades. Nuevas estrategias de intervención en dispositivos de inclusión socioeducativa y laboral para jóvenes en Argentina (2008-2016)

movilizado a los gobiernos del mundo. En Argentina, esta tendencia comenzó a tomar fuerza en la década del noventa en el contexto de un programa de reformas estructurales. Una amplia gama de propuestas de intervención, que incluyó tanto medidas pasivas (asistencia dineraria al desempleo sin contraprestación) como activas (acotadas a subsidios a cambio de la movilización a través de alguna actividad), buscó incidir tanto en la formación profesional como en la demanda de empleo hacia los jóvenes y, en menor medida, corregir asimetrías de oportunidades y facilitar canales de acceso a una mejor inserción laboral (Lasida, 2004; Salvia y Tuñón, 2003b; Salvia, 2008).

También adquirieron relevancia en esa década reformas estructurales que propiciaron el aumento de los niveles de escolarización de los jóvenes, bajo el argumento de que el problema de integración de este grupo etario tenía como principal fuente la deficitaria calidad educativa. Pero este proceso estuvo acompañado de menores niveles de inversión y una enorme fragmentación del sistema (Tedesco y Tenti Fanfani, 2002; Riquelme, 2006; Tiramonti, 2004; Salvia, 2008).

Con el nuevo siglo comienza a desarrollarse un cambio de perspectiva en el diagnóstico de las causas del desempleo juvenil, ya que las argumentaciones referidas al déficit de capital humano o del mercado laboral poco flexible empiezan a perder fuerza frente a una mirada más amplia y estructural. Así, la escolaridad ya no solo formará parte de las políticas educativas sino también de las de empleo, al promover la mayor permanencia de los jóvenes en la escolaridad, sea a través de la educación regular o bien a través de vías alternativas como, por ejemplo, la educación de adultos. Se responde de este modo a una perspectiva que valoriza la escolaridad formal y la adquisición de competencias transversales, tanto para la vida ciudadana como para la laboral. Por su parte, las políticas que articulan educación y trabajo con eje en la activación ${ }^{1}$ se apoyan en intervenciones

\footnotetext{
1 Si bien existen diferentes perspectivas sobre el paradigma de la activación en las políticas de empleo, algunas están ligadas a la responsabilidad individual de incorporarse al mercado laboral, mientras que otras pretenden adaptar la fuerza de trabajo a los requisitos del mercado y así mejorar su empleabilidad (Pérez y
} 
De políticas y subjetividades. Nuevas estrategias de intervención en dispositivos de inclusión socioeducativa y laboral para jóvenes en Argentina (2008-2016)

que brindan herramientas para el acceso y permanencia en el mercado, y en la creación de servicios de empleo cuya clave es la orientación personalizada (Pérez y Brown, 2014; Partenio, 2015).

Así, una de las cuestiones más novedosas de estas políticas es que empiezan a considerar la construcción de las subjetividades de los jóvenes e intentan mediar en sus disposiciones hacia el trabajo. Esto se ve en varios dispositivos que incluyen e integran procesos de orientación socio-laboral y personal. Cuando el trabajo sobre las subjetividades se convierte en estrategia de inclusión se proponen acciones con un alto grado de personalización y de acompañamiento, que propenden al desarrollo de actitudes, valores, motivaciones y expectativas, y a la recuperación de la autoestima (Jacinto, 2006; Jacinto y Millenaar, 2009).

Se comprende que desarrollar la subjetividad de los jóvenes no puede reducirse a la capacitación o formación, sino que éstas deben estar acompañadas por otras acciones tales como la tutoría permanente, la orientación y el seguimiento. Esto es consecuente con la investigación disponible, que señala que la orientación socio-laboral puede contribuir a procesos más amplios de subjetivación (Dávila y Honores, 2003; Abdala, Jacinto y Solla, 2005). En este marco, algunas experiencias latinoamericanas proponen combinar instituciones y diferentes actores que apoyen el desarrollo de evaluaciones y planes de acción personales para promover la reflexibilidad individual y la construcción de soportes institucionales y colectivos. Por el contrario, otras apelan a un carácter instrumental y acotado (cómo hacer un currículum; cómo encontrar trabajo), que brinda poca atención a la necesidad de una fuerte personalización y el lugar central que ocupa la institución formativa en ello (Jacinto, 2010b).

Brown, 2014). Desde esta última mirada, ciertas políticas públicas recientes buscan que su perceptor asuma y se involucre en la construcción de su trayectoria laboral, dado que se considera que los jóvenes desocupados poseen condiciones que pueden ser movilizadas para mejorar su situación. 


\section{Los dispositivos mediadores en la construcción de subjetividades}

Como se ha señalado, es fundamental comprender las mediaciones que existen entre las condiciones estructurales y lo subjetivo, como fuente para el desarrollo de oportunidades y recursos en la gestión de la transición a la vida adulta. Así, resulta de interés recuperar el papel de los dispositivos de inclusión socioeducativa y laboral dirigidos a jóvenes en la construcción de subjetividades.

El concepto de dispositivo, partiendo de la propuesta de Foucault (1984), refiere a una red de relaciones entre instituciones, normas, procesos económicos, sociales, técnicos, y tipos de clasificación de sujetos, objetos y relaciones entre éstos. Un juego de relaciones discursivas y no discursivas, de regularidades cuyo soporte son prácticas, situadas históricamente, que actúan de manera articulada produciendo formas de subjetividad; es decir, inscribiendo en los cuerpos de los sujetos un modo y una forma de ser (Millenaar, 2016b; García Fanlo, 2011). Desde esta perspectiva, los dispositivos son una forma de intervención que pretende reestructurar procedimientos conocidos por o instalados en los sujetos, mediante ciertas formas de aprendizaje y de modificación de "individuos", que implica tanto la adquisición de habilidades como también de ciertas actitudes (Soldano, 2009).

En esta línea, siguiendo también la propuesta de Jacinto (2010a), los "dispositivos de apoyo a la inserción laboral" son intervenciones —enmarcadas o no dentro de políticas públicas- que se proponen explícitamente intervenir para mejorar la inserción laboral de los jóvenes. Estas prácticas son singulares porque emergen en respuesta a acontecimientos y contextos históricos particulares (García Fanlo, 2011) y pueden ser de diversos tipos, conforme a las lógicas de acción que propongan (Jacinto, 2010a): a) retención o terminación de la educación secundaria; b) primer empleo, experiencias de práctica laboral y formación profesional; c) otras experiencias de formación y/o generación de trabajo por cuenta propia o microemprendimientos. 
Frente al reconocimiento de la necesidad y la obligatoriedad de la escuela secundaria, muchos dispositivos - vinculados tanto a políticas educativas como de empleo- apoyan la terminación de la escolaridad de ese nivel. Si bien las políticas de educación secundaria tienen un enfoque amplio y promueven la inclusión generalizada, al mismo tiempo presentan una segmentación. En ello se enmarca la puesta en marcha de dispositivos que tratan de resarcir las carencias históricas del nivel secundario al fortalecer la educación de jóvenes y adultos, al tiempo que darle mayor flexibilidad para responder a múltiples subjetividades.

En lo referido a las estrategias más directamente vinculadas con la inserción laboral, cabe señalar la dimensión de los alcances y precisiones sobre cómo se tienen en cuenta, tanto a nivel de políticas públicas como a nivel microinstitucional, los condicionantes que enmarcan las biografías y los propios jóvenes, que desarrollan la gestión de sus trayectorias (Jacinto, 2010a).

Recuperando la perspectiva de la sociología fenomenológica de Alfred Schütz, centrada en el análisis de la subjetividad y definida como el "complejo de percepciones, vivencias y experiencias del actor ocurridas en un contexto intersubjetivo del cual es productor y usuario" (Soldano, 2009, p. 238), se genera un doble juego en el que los dispositivos de formación y empleo inciden sobre las subjetividades -opiniones, acciones y prácticas-, al tiempo que sus protagonistas negocian sobre y con estas estrategias de intervención.

Así, desde los dispositivos se buscaría inscribir en los sujetos -mediante diferentes experiencias y estrategias - un conjunto de prácticas, saberes y comportamientos que se suponen útiles en la construcción de sus trayectorias de inserción. Desde el lado de los individuos, la incidencia daría cuenta de los modos en que se apropian y motivan a partir de estas experiencias y estrategias, la forma en que las utilizan para procurarse otros recursos tales como capital social, apoyo económico, participación social, entre otros (Jacinto, 2010a). En efecto, interesa analizar las estrategias de intervención en las subjetividades en las dos dimensiones: sobre los jóvenes - a partir de lo propuesto por los dispositivos- y desde los jóvenes, lo negociado y (re) significado por los protagonistas. 
De políticas y subjetividades. Nuevas estrategias de intervención en dispositivos de inclusión socioeducativa y laboral para jóvenes en Argentina (2008-2016)

Para este artículo se seleccionaron dos dispositivos que trabajan sobre las subjetividades de los jóvenes. ${ }^{2}$ Como primer caso se presenta el Programa Jóvenes con Más y Mejor Trabajo (PJMMT) —implementado en La Matanza, Provincia de Buenos Aires-, que explícitamente busca, a través de sus diferentes prestaciones, aportar a la inclusión social y laboral mediante estrategias de desarrollo de la subjetividad, en especial a través de la orientación para el trabajo. Como segundo caso se analiza un Centro de Formación Profesional (CFP) Partido de Tigre, Provincia de Buenos Aires- que, de manera articulada con otras instituciones de su zona de actuación, plantea atender necesidades formativas de jóvenes y adultos, a partir de una serie de estrategias que articulan experiencias educativas y laborales que buscan incidir en las valoraciones y sentidos de sus participantes.

$\mathrm{Si}$ bien se originan en diferentes instituciones y proponen alcances disímiles, ambas intervenciones permiten reflexionar sobre los soportes subjetivos desarrollados para mejorar las oportunidades de los jóvenes. Los dispositivos muestran estrategias diferenciadas de intervención, generadas por las instituciones y gestionadas por sus equipos técnicos/referentes, así como también el desarrollo de valoraciones, opiniones y prácticas por parte de los propios jóvenes que participan de ellos. De ese modo, se parte de la idea de abordar desde ambas dimensiones de análisis- los procesos de subjetivación que entrañan las propuestas, sin pretender comparaciones.

Los datos empíricos fueron construidos siguiendo estrategias cualitativas. En el caso del PJMMT, el trabajo de campo consistió en la realización de entrevistas en profundidad, tanto a funcionarios políticos y equipos técnicos del programa como a jóvenes participantes. Asimismo, se recurrió al análisis

\footnotetext{
${ }^{2}$ La selección se realizó a partir del trabajo empírico desarrollado en el marco de dos tesis doctorales en curso. La primera, de Eugenia Roberti, tiene por objetivo principal comprender la configuración de las trayectorias formativo-laborales de jóvenes participantes del PJMMT durante el período 2008-2016. La segunda, de Alegrando Burgos, estudia novedosas prácticas de educación y formación para el trabajo en el marco de políticas sociales que se proponen garantizar la inclusión educativa y laboral durante el período 2008-2016.
}

Revista Páginas de Educación. Vol. 9, Núm. 2 (2016) ISSN: 1688-5287; e-ISSN: 1688-7468 
De políticas y subjetividades. Nuevas estrategias de intervención en dispositivos de inclusión socioeducativa y laboral para jóvenes en Argentina (2008-2016)

documental, sistematizando información proveniente de resoluciones ministeriales y documentos programáticos. En el caso del CFP, se trabajó a partir de la realización de entrevistas en profundidad a estudiantes, egresados y directivos del centro.

\section{ENTRE LAS POLÍTICAS DE EMPLEO Y LAS ESTRATEGIAS DE SUBJETIVACIÓN: EL PROGRAMA JÓVENES CON MÁS Y MEJOR TRABAJO (LA MATANZA)}

A comienzos del siglo XXI las intervenciones públicas en materia de empleo juvenil se reconfiguran mediante el diseño de políticas activas. En este contexto surge —en el año 2008- el Programa Jóvenes con Más y Mejor Trabajo, que proporciona en forma simultánea prestaciones de seguridad económica y componentes de políticas activas de empleo, con el objetivo de generar oportunidades de inclusión socio-laboral. Este dispositivo, ideado e implementado por el Ministerio de Trabajo, Empleo y Seguridad Social de la Nación (MTEySS), se dirige a jóvenes de entre 18 y 24 años que estén desocupados y no hayan finalizado sus estudios secundarios. El programa brinda un conjunto integrado de prestaciones para apoyar la construcción e implementación de un proyecto formativo-ocupacional que permita a los participantes identificar el perfil profesional en el que deseen desempeñarse, finalizar su escolaridad obligatoria, realizar experiencias de formación o prácticas calificantes en ambientes de trabajo, iniciar una actividad productiva de manera independiente o insertarse en un empleo (MTEySS, 2008).

El análisis del caso que se presenta a continuación se propone comprender cómo esta política de empleo busca incidir sobre las subjetividades juveniles. En especial, se pone foco en la orientación para el trabajo, como parte de las iniciativas novedosas de intervención que inauguran las recientes políticas de juventud. Con esta finalidad, se indaga el modo en que se configura la gestión cotidiana del PJMMT en una jurisdicción específica - La Matanza, Provincia de Buenos Aires - teniendo en cuenta las siguientes dimensiones: por un lado, las 
estrategias de intervención que se formulan desde la normativa y durante la implementación que realizan los equipos técnicos del PJMMT; por otro lado, los sentidos y estrategias que los jóvenes participantes despliegan al interior del programa. Se sostiene que entre ambos niveles analíticos surgen tensiones y desacoples que resignifican la manera en que la orientación socio-laboral contribuye a los procesos más amplios de subjetivación.

\section{Las estrategias de intervención institucional sobre las subjetividades: distancias entre la normativa y la implementación programática}

El PJMMT se plantea como una respuesta institucional diferente, que hace hincapié en la activación de las juventudes. En primer lugar, como advierten las entrevistas al equipo técnico, el aspecto central es que los jóvenes deben gestionar alguna actividad para recibir un incentivo económico (no remunerativo) a cambio. De este modo, se establece un distanciamiento entre el plan y el programa, donde el estímulo económico está condicionado al cumplimiento de ciertos requisitos. Se contrapone así el modelo de asistencia social, que percibe al beneficiario como "un sujeto pasivo", con un modelo que concibe a los jóvenes como "sujetos de participación, de opinión y de toma de posición" (director del PJMMT La Matanza, comunicación personal, septiembre de 2014).

La idea es convencerlos de que sigan estudiando y se capaciten. Este no es un plan en el que te pagan por hacer nada, no es el "plan de los vagos", como lo suelen nombrar en la tele [...] Enseñarles a ellos que no es un plan en que el Estado les va a tener que pagar siempre, sino que es una posibilidad de una capacitación y una beca para que ellos progresen. Es una beca por un lapso de tiempo- para que le saquen jugo, puedan progresar y tener un mejor perfil de ellos mismos... presentar un currículum con un montón de experiencias de cursos y de prácticas laborales (subcoordinadora del PJMMT La Matanza, comunicación personal, agosto de 2015). 
Se plantea entonces un desafío a las mediaciones institucionales, que deben diseñar estrategias de intervención que actúen sobre las subjetividades. En efecto, se visualiza la problemática de la inserción laboral juvenil como una responsabilidad individual, conceptualizada por el equipo técnico local en términos de falta de cultura de trabajo, poco disciplinamiento, déficit de saberes. A esto se suma la particularidad de la edad, asociada con la ausencia de proyección, escasa motivación e interés. Surge así un énfasis en la orientación como un elemento clave vinculado a las políticas activas de empleo, que busca incidir sobre las actitudes y disposiciones de los jóvenes hacia el trabajo.

De este modo, se comienza a configurar un cambio en los ejes conceptuales para abordar los problemas de la inserción laboral de los jóvenes: de las "necesidades de capacitación" hacia las "disposiciones al empleo". Vinculado a una lógica de activación, la orientación pasa a ser una de las medidas clave de las políticas de empleo, de la mano de las concepciones basadas en "dar herramientas para el manejo de la propia trayectoria" (Jacinto, 2010b, p. 139). Este componente se fue consolidando dentro de la línea programática analizada, y aparece como un tipo de intervención que abarca desde un carácter puramente instrumental hasta la búsqueda de una orientación más integral, por medio de un fortalecimiento de las subjetividades. En efecto, no solo aborda aspectos ligados con la formación actitudinal, sino que también busca incidir sobre otros aspectos subjetivos centrados en el fortalecimiento de la autoestima y el desarrollo de habilidades comunicacionales e interaccionales para el mundo laboral (Jacinto y Millenaar, 2012; Gutiérrez y Assusa, 2016).

El Curso de Introducción al Trabajo (CIT) es el único componente obligatorio, al constituirse en el eje y la puerta de entrada del programa. Cada participante inicia su vinculación a través de un proceso de orientación e inducción al mundo del trabajo, en el que se desarrollan aprendizajes vinculados con la elaboración de un currículum vitae, la disposición de herramientas para la búsqueda de empleo, el buen desempeño en entrevistas laborales; así como 
De políticas y subjetividades. Nuevas estrategias de intervención en dispositivos de inclusión socioeducativa y laboral para jóvenes en Argentina (2008-2016)

también conocimientos sobre derechos y deberes del mercado de trabajo e, incluso, la configuración de un proyecto formativo-ocupacional. Este último módulo adquiere especial importancia en los objetivos programáticos, al impulsar la activación del joven, quien debe hacer una reconstrucción de sus experiencias laborales y saberes adquiridos para, a partir de allí, configurar un perfil ocupacional adecuado para insertarse al mercado de trabajo. Este modo de intervención implica la realización de un proceso de autoconocimiento o autoanálisis, en el que los participantes desarrollan estrategias de evaluación sobre sí mismos para identificar sus fortalezas y asumir sus debilidades personales, al tiempo que tiende una mirada reflexiva sobre las oportunidades y limitantes del entorno.

Como apunta el equipo técnico a cargo de la ejecución de este componente, durante el curso se busca dar "voz a los jóvenes", poner en valor saberes, competencias y trayectos laborales invisibilizados —muchas veces ocultos-al desarrollarse al interior del propio hogar:

Trabajar esas cuestiones para que el joven proyecte un trayecto posible en su contexto productivo y sus posibilidades reales también, de acuerdo a las capacidades y trayectorias que haya realizado. [...] La formación de un proyecto ocupacional permite mejorar la autoestima de los jóvenes; llegan al programa diciendo que "no saben hacer nada", "les viene bien cualquier trabajo". Es un espacio donde el joven puede concebir los saberes incorporados, previos, ya que muchas veces trabajan con sus padres y no conciben esa tarea como un trabajo (miembro del equipo técnico del PJMMT, comunicación personal, mayo de 2015).

De acuerdo con la normativa, la formulación del proyecto ocupacional implica un recorrido cuyo punto de llegada es que cada joven pueda definir —en función de sus expectativas - las metas y la ejecución posterior de un itinerario que busque 
De políticas y subjetividades. Nuevas estrategias de intervención en dispositivos de inclusión socioeducativa y laboral para jóvenes en Argentina (2008-2016)

hacer puentes con el trabajo. Esto conlleva la realización selectiva o acumulativa de otras prestaciones, en concordancia con el carácter integral que subyace a la política. Así, esta estrategia de subjetivación sienta sus bases en un diagnóstico orientado por la noción de individualización. Precisamente, el programa impulsa la gestión de las trayectorias por parte de los jóvenes, quienes deben configurar sus recorridos de acuerdo a los propios contextos, experiencias e intereses. Si bien las prestaciones se presentan como acciones integradas, las trayectorias que despliega cada uno de los participantes son particulares. Por consiguiente, es el joven quien carga de un sentido al "programa institucional", al delinear un itinerario específico por sus distintos componentes:

\begin{abstract}
Es lavarles la cabeza para que ellos caigan en que esta es su vida, si no sale de ellos prepararse y luchar, no se puede. El Estado no puede hacer todo. Entonces es para que tomen conciencia de que esto es exclusivamente para ellos. No es venir y cumplir las horas, porque las tenés que cumplir para cobrar. Es venir y cambiar tu mentalidad, ponerte vos mismo tu meta y tu proyecto con la ayuda de los talleristas (subcoordinadora del PJMMT La Matanza, comunicación personal, agosto de 2015).
\end{abstract}

En este proceso cada joven cuenta con la asistencia de un tutor, que es responsable de acompañarlo y derivarlo a las distintas actividades a lo largo de su permanencia en el programa. Ahora bien, a diferencia de lo establecido en la "letra" del PJMMT, en la implementación el CIT aparece como la única instancia colectiva donde el equipo técnico logra un mayor acercamiento al joven. Luego de ese módulo introductorio, los participantes desarrollan un trayecto más individualizado y se diseminan por un conjunto variado de instituciones que participan como prestadoras de los servicios de empleo, lo que dificulta una intervención personalizada. Esta debilidad en los procesos de acompañamiento se agudiza con la masificación del programa, al conducir a que adquieran una mayor 
De políticas y subjetividades. Nuevas estrategias de intervención en dispositivos de inclusión socioeducativa y laboral para jóvenes en Argentina (2008-2016)

relevancia las tareas administrativas frente a los roles de orientación y contención que deberían realizar los tutores.

\section{Redefiniendo las estrategias de orientación para el trabajo: diversidad de sentidos y trayectorias desde los jóvenes}

El énfasis en la individualización se refleja no solo en los diversos caminos que desarrollan los jóvenes al interior del programa, sino también en los múltiples sentidos que complejizan los objetivos programáticos. Si en la normativa del PJMMT se pone foco en la orientación -a través de la conformación de un proyecto formativo en pos de insertarse al mercado laboral-, en el proceso de implementación las trayectorias electivas que desarrollan los jóvenes vislumbran un agenciamiento que, lejos de configurar un perfil ocupacional, toman por fundamento un conjunto de "lógicas no programáticas": afectivas (la sociabilidad), de (pro)actividad ("hacer algo") o de inserción laboral (con base en tramas institucionales).

Con relación a la lógica afectiva, es importarte señalar que entre los sentidos no previstos por las acciones programáticas se encuentra la configuración de redes sociales o sociabilidades juveniles. Precisamente, una novedad del CIT es que propone una instancia de participación grupal donde el joven se contacta con sus pares. ${ }^{3}$ Estos espacios de encuentro, de creación de amistades y comprensión de las mismas problemáticas que los afectan, impulsan a los participantes a continuar el recorrido programático, con menos probabilidades de deserción:

\footnotetext{
${ }^{3}$ Es a través de ese módulo que cada beneficiario define qué otras prestaciones tomar y construye así su proyecto formativo-ocupacional como mecanismo de inclusión socio-laboral. Este curso introductorio está compuesto por cuatro talleres: Proyecto Formativo y Ocupacional; Derechos y Deberes de los trabajadores; Condiciones de Trabajo y Salud Ocupacional; Alfabetización Digital. Tiene una duración de 130 horas, a lo largo de tres meses, y cuenta con el acompañamiento de un tallerista - a cargo de su dictado— que ayuda a los participantes a detectar estrategias adecuadas para planificar y desarrollar un camino de búsqueda, formación y acceso al empleo.
}

Revista Páginas de Educación. Vol. 9, Núm. 2 (2016) ISSN: 1688-5287; e-ISSN: 1688-7468 
Fui y me anoté, empecé en la sede de Catán, ahí hice los primeros dos meses y, bueno, después de eso tenía que buscar un curso para seguir cobrando. Como acá había un montón de cursos, con mi grupo de amigos, que habíamos hecho ahí, buscamos en los alrededores. Encontré el de operador de PC y ahí empecé acá (ex beneficiaria del PJMMT, comunicación personal, diciembre de 2015).

En la lógica de (pro)actividad se observa que, lejos de ser la "inserción laboral de calidad" - como sugiere la normativa del PJMMT - lo que orienta el ingreso al programa y la realización de nuevas prestaciones, prima - por el contrario- un impulso a "hacer algo", que abarca desde la búsqueda de herramientas para la vida hasta una inversión del tiempo por medio de la realización de actividades, que distan de contemplar un itinerario de acceso al empleo. A través de los relatos se devela que la (pro)actividad implica muchas veces un rumbo sin planificación u orientación, que entra en tensión con el itinerario planeado en el proyecto formativo-ocupacional, a partir del cual se orientan las prestaciones realizadas. En este punto es interesante vislumbrar la lógica que está detrás del pasaje que algunos jóvenes realizan por el programa, ya que otorgan nuevos significados a su participación.

—¿Qué te lleva a hacer tantos cursos? ¿Qué es lo que esperás de ellos?

-Al principio era por lo económico. Después me di cuenta de que, si me quedo con una sola cosa, sé hacer solo una cosa y para otras cosas no voy a servir. Yo busco cursos adonde puedo sacar provecho... no solamente conseguir laburo de eso, sino también que me ayude a mí, en mi vida cotidiana. Donde soy un desastre, se puede decir, me ayudan un poco, me moldean y salgo mejor. Si voy a la casa de mi abuela y tiene un problema eléctrico, como soy electricista puedo ayudar y no tiene que llamar a otra persona 
De políticas y subjetividades. Nuevas estrategias de intervención en dispositivos de inclusión socioeducativa y laboral para jóvenes en Argentina (2008-2016)

(beneficiario del PJMMT, comunicación personal, noviembre de 2015).

Por último, se encuentra el grupo de jóvenes que despliega trayectorias de inserción laboral al interior de las instituciones que brindan servicios programáticos. A través de estrechos vínculos institucionales, los participantes logran incorporarse a circuitos donde se desarrollan relaciones cara a cara, que no se ven interrumpidas con la masividad del programa. El establecimiento de lazos más fuertes permite a los entrevistados construir un puente directo con el trabajo, que se encuentra amparado por su mayor capital cultural y social. Así, la oportunidad de inserción laboral se efectiviza bajo esta lógica e, incluso, para muchos jóvenes significa un primer trabajo "en blanco":

Y lo que más me gustó fue que a través de esto brindan oportunidades a los jóvenes, oportunidades para seguir estudiando o mismo para tener mi primer empleo (beneficiaria del PJMMT, comunicación personal, octubre de 2015).

En suma, a partir del análisis de este caso se despliega el carácter paradójico de un proceso donde la subjetividad de los jóvenes emerge como una madeja biográfica susceptible de ser intervenida, orientada y regulada por diversas instituciones, a la vez que se realizan llamados al ejercicio de la responsabilidad individual: cada uno de los participantes tiene que explorar y conformar su propio proyecto ocupacional.

Sin embargo, como se vislumbra en la última de las lógicas, el éxito de las estrategias de intervención del PJMMT depende de que estas involucren un soporte personalizado y permanente que acompañe las acciones integrales de socialización laboral, formación profesional y terminalidad educativa que desarrollan los jóvenes, con el objetivo de que estos pasajes no sean efímeros 
sino, por el contrario, que comprendan una estrategia sostenible de inserción socio-laboral más inclusiva.

\section{LA FORMACIÓN PARA EL TRABAJO DE JÓVENES Y ADULTOS DE SECTORES SOCIALES BAJOS: CENTRO DE FORMACIÓN PROFESIONAL (TIGRE)}

El Centro de Formación Profesional ${ }^{4}$ que se analiza fue fundado en el año 1989 en un municipio del norte del conurbano de la Provincia de Buenos Aires. Este centro brinda educación y formación para el trabajo a jóvenes y adultos de un barrio de Tigre que se caracteriza por un elevado desempleo y niveles altos de informalidad. Brinda cursos de carpintería, soldadura, tornería, diseño de moda, gastronomía (restaurant 1 y 2), panadería, informática (administración comercial y diseño gráfico), reparación de PC, diseño de páginas web, auxiliar de familia con especialización en adultos mayores, operador socio-comunitario, inglés y turismo. Algunos de los cursos se repiten de un año para el otro, según la demanda, y se dictan dos o tres veces por semana. Los títulos que otorga son certificados por la Dirección General de Cultura y Educación de la Provincia de Buenos Aires.

En este segundo caso se recurre también a las dos dimensiones analíticas referidas a las estrategias de intervención en las subjetividades. Se presenta, por un lado, la incidencia que tienen las estrategias llevadas a cabo por el CFP en las trayectorias educativas y laborales en la construcción de subjetividad de los jóvenes y adultos participantes. Por otro lado, el modo en que los sujetos se apropian y hacen uso de esas estrategias. Asimismo, se considera la interrelación de formación, educación y derechos sociales como uno de los ejes vertebrales que orientan el accionar de este dispositivo en pos de la inclusión social.

\footnotetext{
${ }^{4}$ El CFP forma parte de la Red El Telar y la Red de Formación Laboral de Cáritas San Isidro (Provincia de Buenos Aires). Además, participa en una red barrial de instituciones locales (centros de salud, escuelas primarias, jardín de infantes, centro integral comunitario, departamental de policía y delegación municipal) para articular de forma integral una mejor atención a las necesidades y demandas de los jóvenes y adultos.
} 


\section{Las estrategias de intervención sobre las subjetividades: puntos críticos}

Como se señaló anteriormente, el rol de las instituciones no puede limitarse solo a favorecer el acceso a los sistemas de formación y educación, sino que se torna imperioso considerar aspectos subjetivos y de empoderamiento de los sujetos para garantizar una participación activa en la sociedad civil. En este sentido, en los últimos años, los Centros de Formación Profesional se proponen objetivos que trascienden la formación profesional específica para responder a las necesidades de la población que asisten, a partir de una formación integral (Jacinto, 2015; Millenaar, 2016a). Por un lado, se reconocen las desigualdades sociales y educativas que sufren estos jóvenes y adultos; por otro lado, a las demandas de competencias para el trabajo proponen una formación más amplia, que incluye aspectos actitudinales, comunicacionales y sociales.

El CFP que se presenta en este caso es una institución de origen socialcristiano con una trayectoria de 27 años en el terreno de la formación para el trabajo y la educación. A partir de los testimonios de los directivos, se desprenden como prioritarios dos puntos críticos en los que es necesario desplegar estrategias de intervención sobre las subjetividades ante la situación socioeconómica y educativa de los jóvenes y adultos.

Un punto crítico se asocia con la tarea de aportar para que los jóvenes logren trayectorias laborales estables y en mejores empleos. Esto deviene de considerar tanto la situación del hogar de origen como la propia experiencia de los jóvenes y adultos. Según los directivos, la trayectoria laboral de los padres revela trayectorias signadas por la informalidad y alta rotación por trabajos poco calificados y remunerados. Los padres en su mayoría se insertan en los segmentos más precarios del mercado de trabajo, que abarca diversas actividades laborales como changas, venta ambulante, etc. Frente a la pérdida reciente del empleo o la rotación constante de los padres, la insuficiencia de ingresos percibidos o la ausencia de acceso a cobertura en salud, los jóvenes ingresan al mercado de trabajo de forma prematura como una estrategia de supervivencia de 
De políticas y subjetividades. Nuevas estrategias de intervención en dispositivos de inclusión socioeducativa y laboral para jóvenes en Argentina (2008-2016)

las familias frente a circunstancias adversas. Dentro de este escenario, el acceso de los jóvenes y adultos al mercado de trabajo se concreta en empleos inestables, poco calificados y en su mayoría informales (remisería, quiosco, restaurant, caja de supermercado, servicio doméstico...), que no permiten acumular saberes y experiencias en un rubro $u$ oficio determinado.

Ante esta realidad, la estrategia de intervención desplegada por el CFP incluye el estímulo a la comunicación y la participación social de los jóvenes y adultos, al hacerlos reflexionar sobre sus derechos como individuos, ciudadanos y trabajadores. Este componente reconoce la importancia actual de las competencias interactivas y sociales en los sujetos, para que puedan empoderarse, desarrollar una identidad propia y ganar autonomía para la inserción en el mercado del trabajo. Se busca la recuperación de la autoestima a partir de un trabajo que rescata los saberes y experiencias previas, así como las expectativas y estrategias a futuro que permitan superar el desempleo y las trayectorias laborales inestables. Las entrevistas con los directivos evidencian que los jóvenes y adultos no solamente trabajan por fuera de lo pautado en el derecho laboral, sino que además desconocen en muchos casos la legislación vigente y los derechos laborales. Este es otro aspecto clave en el que se trabaja fuertemente desde el CFP.

Otro de los puntos críticos que deben enfrentarse es que, en su mayoría, los jóvenes y adultos representan la primera generación que cursa y finaliza el nivel secundario. Por ende, son individuos cuyas familias no cuentan con un considerable historial educativo. En términos generales, los estudiantes no habían finalizado los estudios secundarios. Esto llevó al CFP a desarrollar una estrategia de intervención socio-educativa: de allí su vínculo con una sede del Plan FinEs ${ }^{5}$ en

\footnotetext{
${ }^{5}$ El Plan de Finalización de Estudios Primarios y Secundarios tiene alcance nacional, depende del Ministerio de Educación y Deportes de la Nación, en articulación con los ministerios de Educación provinciales. En el año 2008 se plantea una primera etapa, que ofrece la posibilidad de obtener el título secundario a jóvenes y adultos que hayan cursado el último año de la secundaria o polimodal y adeuden materias, sin haber alcanzado el título. En los años siguientes se proponen acciones para aquellos que no iniciaron o bien no finalizaron el nivel primario o secundario. Se suma a la oferta regular de la Educación de Jóvenes y Adultos.
} 
De políticas y subjetividades. Nuevas estrategias de intervención en dispositivos de inclusión socioeducativa y laboral para jóvenes en Argentina (2008-2016)

un barrio cercano al que se ubica el centro, para articular formación para el trabajo con terminalidad educativa en el nivel secundario. Esta articulación —que no tiene carácter institucional formal, sino que responde a las "tramas" institucionales no formales que se tejen para responder a las necesidades de los sujetos - tiene como fin ampliar el acceso a la educación como un derecho social, al incorporar una lógica de recreación de capacidades y de empoderamiento de los sujetos que procure cambiar su imagen vinculada con el fracaso y el abandono escolar. Las palabras de la directora del CFP dejan en claro el complejo escenario:

Recibimos en el Centro personas que han sido derrotadas por el sistema, que los expulsó. Muchos llegan y necesitan capacitarse porque no tienen trabajo y necesitan tener una herramienta. En su mayoría son pibes y adultos desfavorecidos con una condición económica muy pobre, con situaciones muy precarias de laburo (directora del CFP, comunicación personal, junio de 2015).

Así, las estrategias de intervención sobre las subjetividades son complementarias y se refuerzan recíprocamente. Consisten en la realización de acciones de orientación laboral, de capacitación y de espacios que apoyan la terminalidad de los niveles formales del sistema educativo.

\section{Fortalecimiento de competencias socio-laborales y educativas, y su incidencia en la subjetividad desde los jóvenes y adultos}

La situación de los jóvenes y adultos de sectores sociales bajos llevó a la ampliación y diversificación de los Centros de Formación Profesional y sus estrategias. Muchas de ellas presentan enfoques novedosos que implican acciones pedagógicas, psico-sociales y de formación. Así, conjuntamente con los objetivos de inserción laboral —o al menos de progreso de la situación de los jóvenes y adultos - los centros desarrollan otros objetivos centrados en el fortalecimiento de la subjetividad y el empoderamiento, que apunta a la integración 
De políticas y subjetividades. Nuevas estrategias de intervención en dispositivos de inclusión socioeducativa y laboral para jóvenes en Argentina (2008-2016)

social. Ahora bien, cabe preguntarse: ¿cuáles son los significados o sentidos de los jóvenes y adultos con respecto a las estrategias desarrolladas por el CFP en torno a sus subjetividades? Se presenta a continuación la voz de los jóvenes y adultos entrevistados, quienes expresaron una alta valoración de estas estrategias desarrolladas.

En algunas entrevistas se hace referencia al CFP y a los cursos como espacio de socialización y como un instrumento útil. Por un lado, la sociabilidad con pares les aporta trabajar con otros con experiencias similares, al tiempo que obtener informaciones sobre el mercado de trabajo. Por otro, también se valoriza la construcción de amistades con los profesores. Asimismo, en algunas entrevistas se manifiesta que el lazo social que se construye entre los actores es importante, además, para promover la organización de microemprendimientos o como una red de relaciones, tal como expresa un egresado:

Hago lo que es reparación, programas, las instalaciones completas de lo que es una PC. Por suerte me llegan muchas computadoras para hacer. Me llegan trabajos de gente conocida o mucho contacto social en el Centro. Aprendí mucho del profesor, no solo en la informática, sino que con la sinceridad se llega a todos lados. Va de boca en boca. Yo arreglé algunas computadoras acá y esa gente me trae otras y otras. $\mathrm{Y}$ gracias a que encontré un grupo excelente de alumnos y profesores (egresado CFP-estudiante Plan FinEs, comunicación personal, setiembre de 2015).

Se fomenta así, desde el CFP, una construcción colectiva entre los sujetos. El acompañamiento de profesores y de grupos de pares tiene una fuerte incidencia en la subjetividad de los jóvenes y adultos, ya que los posiciona con mayor fortaleza frente a la adversidad. Sin embargo, la organización de microemprendimientos requiere de ciertas condiciones y saberes muchas veces no presentes por la endeblez de las competencias generales y la limitada red de 
De políticas y subjetividades. Nuevas estrategias de intervención en dispositivos de inclusión socioeducativa y laboral para jóvenes en Argentina (2008-2016)

relaciones sociales, a lasque se agrega, en el caso de los jóvenes, la escasa experiencia.

Desde el punto de vista de los participantes, la estrategia centrada en la acumulación de saberes, la estimulación de la comunicación y la participación social tiene una incidencia positiva que redunda en una mayor "valoración de sí mismos". En muchos testimonios se expresa la búsqueda de un proyecto personal que involucra al empleo en función de las propias potencialidades y el valor de los saberes previos y actuales en la construcción de la trayectoria. En otro orden, en los relatos de los jóvenes y adultos se valora ampliamente la palabra de profesores y directivos en el reconocimiento de la educación y el trabajo como un derecho social, al incorporar una lógica de recreación de capacidades y de empoderamiento de los sujetos, en especial en torno a los derechos laborales:

Obvio que me gustaría trabajar de todo lo que aprendí. Porque antes yo no sabía nada y ahora sé un montón de cosas. Cuando uno sabe algo que le gusta, lo ve de manera diferente. Y sí, me gustaría trabajar de lo que aprendí. No sé muy bien en qué, pero me imagino en una empresa de oficinista, trabajando en el Excel para una empresa. Estaría re bueno (egresada CFP-egresada de escuela secundaria común, comunicación personal, noviembre de 2015).

Por último, la estrategia socio-educativa se asocia con el fortalecimiento de las capacidades de aprendizaje y la terminalidad de estudios secundarios a través del Plan FinEs. Se apuesta a construir un espacio áulico que contribuya a generar una experiencia de trabajo colectiva y de intercambio entre los cursos del centro y otras instituciones del barrio. La cercanía barrial y el trabajo colectivo fomentan los valores de solidaridad, compañerismo y el acompañamiento mutuo como aspectos claves en la conformación de un grupo de estudio, elemento central para la permanencia y continuidad. Es manifestada reiteradamente la incidencia que tiene 
De políticas y subjetividades. Nuevas estrategias de intervención en dispositivos de inclusión socioeducativa y laboral para jóvenes en Argentina (2008-2016)

esta estrategia de formación colectiva: por un lado, aporta al mejoramiento en el lenguaje, en la comunicación y en las habilidades relacionadas con el aprendizaje; por otro lado, modifica la imagen respecto de las relaciones con el conocimiento:

Me decían que era un cursito y no me importa, yo lo tengo y lo hice. Y así me manejo. Pero nunca dejo de estudiar. Además de que te mantiene la mente ocupada, crecés un montón. $Y$ hoy me di cuenta de que sé manejarme y hablar con las personas. Sé desenvolverme y todo eso lo aprendés leyendo (egresada CFPegresada Plan FinEs, comunicación personal, noviembre de 2015).

La estrategia integral que combina la formación para el trabajo y la participación en el plan FinEs establece dos dimensiones que inciden, pues, en las subjetividades y su desarrollo, tanto en el plano laboral como para la ciudadanía. Se podría argumentar que el CFP intenta contribuir en esta tarea a partir de la ampliación en el acceso de los jóvenes y adultos al trabajo y la educación como un derecho social. Sin embargo, cabe preguntarse: ¿es suficiente con este tipo de estrategias? ¿Son las estrategias adoptadas por el CFP las más pertinentes para lograr la integración de los jóvenes y adultos de sectores sociales bajos?

Tal como se ha esbozado más arriba, los participantes entrevistados expresan una alta valoración de las estrategias que desarrolla el CFP, porque el centro contempla las particularidades de los sujetos que allí asisten, sus trayectorias laborales y educativas previas, así como el contexto socio-familiar, en tanto introduce estrategias que tienen como prioridad reconocer las vicisitudes y necesidades significativas de los jóvenes y adultos. Sin embargo, son dos los elementos que habilitan un cauto optimismo: por un lado, es necesario contar con un acompañamiento poscurso de formación, que apuntale los microemprendimientos a partir del asesoramiento en temas legales hasta la conformación de un sistema de subsidios o préstamos para la compra de 
De políticas y subjetividades. Nuevas estrategias de intervención en dispositivos de inclusión socioeducativa y laboral para jóvenes en Argentina (2008-2016)

equipamientos; por otro lado, el reconocimiento no garantiza por sí solo la restitución de los derechos de los sujetos. Se requiere, además del trabajo del CFP, el acompañamiento de políticas intersectoriales que prevean intervenciones adecuadas, precisas, oportunas y relevantes para apuntar las trayectorias de los jóvenes y adultos.

\section{REFLEXIONES FINALES}

En el estudio de los casos aquí presentados se puede apreciar que un aspecto central que comparten estos dispositivos, que articulan la educación con el trabajo, radica en que ambos desarrollan estrategias de intervención con eje en la subjetividad. En efecto, las estrategias de orientación socio-laboral, formación profesional y terminalidad educativa que impulsan estos dispositivos involucran no solo el desarrollo de competencias para el trabajo, sino que también proponen una formación más integral que incluye aspectos actitudinales, comunicacionales y sociales. No obstante, se distinguen particularidades en los dispositivos presentados.

En el caso del PJMMT, el apoyo a la subjetividad que promueve se efectúa a través de cursos orientación socio-laboral. La activación de los jóvenes aparece aquí como el resultado esperado de las estrategias de intervención institucionales, que oculta un fuerte carácter individualizante: es el joven quien debe configurar su propio proyecto formativo-ocupacional. Como contracara, las relaciones de proximidad y la contención operan como estrategias que hacen no solo al sostenimiento de los jóvenes en el programa, sino que también funcionan como soportes al despliegue de esa subjetividad.

Las estrategias de intervención desarrolladas por el CFP aspiran a brindar soportes institucionales protectores que tienen incidencia sobre aspectos personales y subjetivos. La lógica de recreación de capacidades sociolaborales, el fortalecimiento de los aprendizajes y el empoderamiento de los sujetos aumentan las posibilidades de inclusión educativa y laboral. Sin embargo, son importantes los desafíos que enfrentan los jóvenes y adultos de 
nuestro tiempo frente a los crecientes procesos de individualización de las trayectorias y el aumento de las desigualdades, que habilitan un cauto optimismo respecto de la ampliación y efectivización del derecho al trabajo y la educación.

Por último, cabe destacar el rico ejercicio de recuperar las voces de los protagonistas a la hora de desentrañar los procesos de subjetivación propuestos por dichos dispositivos y (re)significados por los participantes. En términos generales, debido a que los testimonios traslucen no solo el entramado de instituciones que se conjugan en dichos procesos, sino también las tensiones entre las estrategias de intervención dispuestas y lo sentidos que adquieren para quienes las vivencian. En términos particulares, por los aportes que pueden brindar — como lo han demostrado diversas investigaciones - a la hora de diseñar e implementar políticas públicas.

\section{REFERENCIAS}

Abdala, E., Jacinto, C. y Solla, A. (2005). La inclusión laboral de los jóvenes: entre la desesperanza y la construcción colectiva. Montevideo: Cinterfor.

Arrow, K. (1973). Higher education as a filter. Journal of Political Economics, 2, 193-216.

Beck, U. (2008). La sociedad del riesgo mundial: en busca de la seguridad perdida. Barcelona: Paidós.

Bourdieu, P. y Passeron, J. C. (1967). Les heritiers. Paris: De Minuit.

Bourdieu, P. y Passeron, J. C. (1972). La reproducción. Elementos para una teoría del sistema de enseñanza. Barcelona: Laia.

Casal, J. (1996). Modos emergentes de transición a la vida adulta en el umbral del siglo XXI: aproximación sucesiva, precariedad y desestructuración. Revista Española de Investigaciones Sociológicas, 75, 295-316.

Castel, R. y Haroche, C. (2001). Propriété privée, propriété sociale, propriété de soi. Entretiens sur la construction de l'individu moderne. Paris: Fayard. 
De políticas y subjetividades. Nuevas estrategias de intervención en dispositivos de inclusión socioeducativa y laboral para jóvenes en Argentina (2008-2016)

Carnoy, M. (1982). Economía y educación. Revista del Consejo Nacional Técnico de la Educación, 8 (40), 36-70.

Dávila, O. y Honores, C. (2003). Capital social juvenil y evaluación programática hacia jóvenes. Última Década, 18, 175-198.

Dubar, C. (1991). La socialisation. Construction des identités sociales et professionnelles. París: Armand Colin Editeur.

Dubar, C. (2002). L'articulation des temporalités dans la construction des identités personnelles: questions de recherche et problèmes d'interprétation. Temporalistes, 1 (44), 2-6.

Dubet, F. (2002). Le déclin de l'institution. París: Le Seuil.

Foucault, M. (1984). El juego de Michel Foucault. Saber y verdad(pp. 127-162). Madrid: Ediciones De la Piqueta.

García Fanlo, L. (2011). ¿Qué es un dispositivo? Foucault, Deleuze, Agamben. A Parte Rei. Revista de Filosofía, 74. Recuperado de http://serbal.pntic.mec.es/ cmunoz11/fanlo74.pdf

Galland, O. (2002). Les jeunes. París: Éditions La Découverte.

Gutiérrez, A. y Assusa, G. (2016). El «problema» de la generación, la «generación» del problema. La producción social del problema de la empleabilidad juvenil en documentos de organismos públicos del mundo del trabajo. Papers Revista de Sociología, 101, 73-95.

Hartog, F. (2003). Régimes d'historicité. Présentisme et expériences du temps. París: LeSeuil.

Herger, N. (2008). Las barreras para la construcción de proyectos de educación y exclusión social de jóvenes pobres en Argentina. Salvia, A. (Comp.). Jóvenes promesas. Trabajo, educación y exclusión social de jóvenes pobres en Argentina (pp.181-204). Buenos Aires: Miño y Dávila.

Jacinto, C. (2006). Estrategias sistémicas y subjetivas de transición laboral de los jóvenes en Argentina. El papel de los dispositivos de formación para el empleo. Revista de Educación, 341, 57-79. 
De políticas y subjetividades. Nuevas estrategias de intervención en dispositivos de inclusión socioeducativa y laboral para jóvenes en Argentina (2008-2016)

Jacinto, C. (2007). Diagnóstico, tensiones y recomendaciones de política en relación a los vínculos entre educación y formación laboral de la población adolescente. Buenos Aires: UNICEF.

Jacinto, C. (2008). Políticas públicas, trayectorias y subjetividades en torno a la transición laboral de los jóvenes. Novick, M.y Pérez Sosto, G. (Coords). El estado y la reconfiguración de las protecciones sociales (pp. 463-498). Buenos Aires: Instituto Torcuato Di Tella-Siglo XXI.

Jacinto, C. (2010a). Elementos para un marco analítico de los dispositivos de inserción laboral de jóvenes y su incidencia en las trayectorias. Jacinto, C. (Comp.). La construcción social de las trayectorias laborales de jóvenes: políticas, instituciones, dispositivos y subjetividades (pp. 15-45). Buenos Aires: Teseo/IDES.

Jacinto, C. (2010b). Veinte años de políticas de formación para el empleo de jóvenes vulnerables en América Latina: persistencia y reformulaciones. Jacinto, C. (Comp.). La construcción social de las trayectorias laborales de jóvenes: políticas, instituciones, dispositivos y subjetividades (pp. 119-144). Buenos Aires: Teseo/IDES.

Jacinto, C. (2015). Nuevas lógicas en la formación profesional en Argentina. Redefiniendo lo educativo, lo laboral y lo social. Perfiles Educativos, 37 (148), 120-137.

Jacinto, C. y Millenaar, V. (2009). Enfoques de programas para la inclusión laboral de los jóvenes pobres: lo institucional como soporte subjetivo. Última Década 30, 67-92.

Jacinto, C. y Millenaar, V. (2012). Los nuevos saberes para la inserción laboral. Formación para el trabajo con jóvenes vulnerables en Argentina.Revista Mexicana de Investigación Educativa, 52, 141-166.

Lasida, J. (2004). Estrategias para acercar a los jóvenes al trabajo. Buenos Aires: RedEtis (IIPE-IDES). 
Longo, M. E. (2008). Claves para el análisis de las trayectorias profesionales de los jóvenes: multiplicidad de factores y de temporalidades. Revista Estudios del Trabajo, 35, 73-95.

Longo, M. E. (2010). Las secuencias de inserción: una alternativa para el análisis de trayectorias laborales de jóvenes. Jacinto, C. (Comp.). La construcción social de las trayectorias laborales de jóvenes: políticas, instituciones, dispositivos y subjetividades (pp. 259-295). Buenos Aires: Teseo/IDES.

Millenaar, V. (2016a). Educación y trabajo en la Formación Profesional. Revista Novedades Educativas, 306, 32-35.

Millenaar, V. (2016b). La Formación Profesional en la Argentina: procesos históricos y lógicas territoriales. Documento en prensa.

Morch, M. (2002). Sistemas Educativos en Sociedades Segmentadas: trayectorias fallidas en Dinamarca, Alemania oriental y España. Revista de Juventud 56 (02), 31-54.

Nicole-Drancourt, C. y Roulleau-Berger, L. (2001).Les jeunes et le travail 19502000. París: PUF.

Partenio, F. (2015). Aprendizaje de calidad y preparación de los jóvenes para el trabajo en Argentina. Montevideo: OIT/Cinterfor.

Pérez, P. y Brown, B. (2014). Políticas de empleo para jóvenes: el programa Jóvenes con y Mejor Trabajo. Pérez, P. y Busso, M. Tiempos contingentes: inserción laboral de los jóvenes en la Argentina posneoliberal (pp. 147-166). Buenos Aires: Miño y Dávila.

Pérez, P. (2008). La inserción ocupacional de los jóvenes en un contexto de desempleo masivo. El caso argentino entre 1995 y 2003. Buenos Aires: Miño y Dávila.

MTEySS. (2008). Resolución N 497/08. Programa Jóvenes con Más y Mejor Trabajo. Recuperado de http://www.trabajo.gov.ar/downloads/programajovenes/res se 2612008 jovenes con mas y mejor.pdf 
Riquelme, G. (2001). Acceso a la educación y formación para el trabajo: quiénes y qué tipo de recursos. Ponencia presentada en el $V$ Congreso Nacional de ASET. Buenos Aires, Argentina.

Riquelme, G. (2006). La relación entre educación y trabajo: continuidad, rupturas y desafíos. Anales de la educación común, 2 (5), 68-75.

Robles, F. (2000). El desaliento inesperado de la modernidad. Molestias, irritaciones y frutos amargos de la sociedad de riesgo. Santiago de Chile: RIL Editores.

Salvia, A. (2008). Introducción: La cuestión juvenil bajo sospecha. Salvia, A. (Comp.) Jóvenes promesas. Trabajo, educación y exclusión social de jóvenes pobres en Argentina (pp. 13-31). Buenos Aires: Miño y Dávila.

Salvia, A. y Tuñón, I. (2003a). Los jóvenes trabajadores frente a la Educación, el desempleo y el deterioro social en Argentina. Buenos Aites: Friedrich Ebert Stiftung.

Salvia, A. y Tuñón, I. (2003b). Jóvenes trabajadores en Cono Sur: desafíos y respuestas. Proyecto PROSUR. Buenos Aires: Fundación Friedrich Ebert.

Soldano, D. (2009). El Estado en la vida cotidiana. Algunos desafíos conceptuales y metodológicos de la investigación sobre política y biografía. Frederic, S. y Soprano, G. (Comps.). Política y variaciones de escalas en el análisis de la Argentina (pp.235-254). Buenos Aires: Prometeo.

Tedesco, J. C. (2008). Democracia y educación. Conferencia Magistral dictada en el $44^{\circ}$ Curso de rectores. Buenos Aires: CONSUDEC.

Tedesco, J. C. y Tenti Fanfani, E. (2002). Nuevos tiempos y nuevos docentes. Buenos Aires: IIPE.

Tiramonti, G. (Comp.). (2004). La trama de la desigualdad educativa: mutaciones recientes en la escuela media. Buenos Aires: Manantial.

Walther, A. y Pohl, A. (2005). Thematic study on policy measures concerning disadvantaged youth. Tübingen:IRIS.

Weller, J. (2007). La inserción laboral de jóvenes: características, tensiones y desafíos. Revista de la CEPAL, 92, 61-82. 